\title{
Atividade antioxidante e compostos fenólicos totais em vinagres
}

\author{
Antioxidant activity and total phenolic compounds in vinegars
}

\author{
Michelle Tieko Kobayashi ${ }^{1}$; Ana Carolina da Silva² \\ ${ }^{1}$ Aluna do Curso de Graduação em Engenharia de Alimentos, Universidade Federal do Triângulo Mineiro, \\ Uberaba, Minas Gerais, Brasil. E-mail: mitikobayashi@gmail.com \\ ${ }^{2}$ Professora do Curso de Engenharia de Alimentos, Universidade Federal do Triângulo Mineiro, Uberaba, \\ Minas Gerais, Brasil. Orcid: http://orcid.org/0000-0001-6272-6147. E-mail: ana.silva@uftm.edu.br
}

\begin{abstract}
RESUMO: O vinagre, ou fermentado acético, conforme a legislação, é o produto obtido por fermentação acética do vinho. É um produto que possui compostos bioativos que funcionam como estimulantes da digestão, tem ação antisséptica, propriedade desinfetante e anti-inflamatória entre outros benefícios à saúde. O presente trabalho teve como objetivos avaliar a atividade antioxidante e determinar o conteúdo de polifenóis de dos vinagres de vinho tinto, vinho branco, maçã e de álcool, de três diferentes marcas comerciais cada. Foram realizadas análises para identificar a atividade antioxidante através dos métodos $\mathrm{ABTS}^{*+}$ e DPPH ${ }^{\circ}$ e o conteúdo de polifenóis, por meio do método de Folin-Ciocalteau. Os vinagres de vinho tinto apresentaram melhores resultados quanto ao teor de compostos fenólicos totais, com média de $516,34 \mathrm{mg} \mathrm{mL}^{-1}$, e atividade antioxidante pelo método ABTS ${ }^{++}$, com média 2215,74 $\mu \mathrm{M}$ de Trolox. Já na análise de $\mathrm{DPPH}^{\circ}$ destacaram-se os vinagres de maçã e de vinho branco com, aproximadamente, 95\% de atividade antioxidante para ambos, independente da marca comercial. Dentre os tipos de vinagres estudados, o de álcool foi o que apresentou os piores resultados de atividade antioxidante e de compostos fenólicos totais. Conclui-se que há diferença entre os tipos de vinagres para os parâmetros analisados e que, deferentes marcas de um mesmo um tipo de vinagre, podem apresentar diferença na composição. De maneira geral, os vinagres de vinho tinto, vinho branco e maçã apresentaram melhores resultados para atividade antioxidante e compostos fenólicos.
\end{abstract}

Palavras-chave: Folin-Ciocalteau, $\mathrm{ABTS}^{\circ}{ }^{+}$, DPPH${ }^{\bullet}$, espectrofotometria, vinagre.

ABSTRACT: Vinegar, or acetic fermented, according to the legislation, is the product obtained by acetic fermentation of wine. It is a product that has bioactive compounds that act as a digestion stimulant, has antiseptic action, disinfectant property and anti-inflammatory among other health benefits. The present work aims to evaluate the antioxidant activity and determine the content of polyphenols of the red wine, white wine, apple and alcohol vinegars, of three different commercial brands each. Analyzes were performed to identify the antioxidant activity through the ABTS ${ }^{\circ+}$ and $D P P H$ methods and the polyphenol content through the Folin-Ciocalteau method. Red wine vinegars showed better results in terms of total phenolic compounds, with $516.34 \mathrm{mg} \mathrm{mL}^{-1}$ average, and antioxidant activity by the ABTS - + method, with $2215.74 \mu \mathrm{M}$ of Trolox average. In the analysis of DPPH • apple and white wine vinegars stood out with approximately $95 \%$ antioxidant activity for both, regardless of the commercial brand. Among the types of vinegars studied, alcohol was the one that presented the worst results of antioxidant activity and total phenolic compounds. It is concluded that there is a difference between the types of vinegar for the analyzed parameters and that, different brands of the same type of vinegar, may present a difference in composition. In general, red wine, white wine and apple vinegars showed better results for antioxidant activity and phenolic compounds.

Keywords: Folin-Ciocalteau, ABTS ${ }^{+}, D P P H$, spectrophotometry, vinegars. 


\section{INTRODUÇÃO}

O vinagre é um produto conhecido há muito tempo e no mundo todo. Os primeiros registros da existência do vinagre datam de 8000 a.C. no Egito antigo, usado como medicamento.

O vinagre é conhecido pelas suas propriedades benéficas ao organismo humano e tem importância na alimentação desde a antiguidade. Este produto era muito utilizado como uma bebida refrescante diluído em água, e como medicamento para tratar disfunções respiratórias, feridas e úlceras, devido às suas propriedades desinfetantes e anti-inflamatórias. Nas epidemias de cólera na Europa era recomendado o uso por sua ação asséptica, na desinfecção e para prevenir possíveis contaminações microbiológicas (RIZZON, 2002).

A palavra vinagre é originada do francês "vinaigre", ou seja, vinho azedo. Este produto é obtido primeiro por uma fermentação alcoólica, na qual ocorre a transformação de glicídios em álcool e seguida por uma fermentação acética. $\mathrm{O}$ álcool obtido na etapa anterior é transformado em ácido acético formando por fim o vinagre (MARQUES et al., 2010).

Para a fabricação do vinagre pode ser utilizada qualquer matéria-prima que tenha sacarídeo, como exemplo, arroz, cana de açúcar, cevada, beterraba, batata, algumas frutas como maçã e uva. Assim, no mercado existe ampla variedade de tipos de vinagre.

Segundo os dados da Associação Nacional das Indústrias de Vinagre (ANAV, 2019), são consumidos no Brasil aproximadamente 170 milhões de litros de vinagre por ano. Cerca de $80 \%$ deste total é referente a vinagres de álcool. Em média, cada brasileiro consome, $800 \mathrm{ml}$ de vinagre por ano. Já nos países europeus e Estados Unidos a média per capita de consumo é cerca de 1,8 litros por ano.

Sobre os aspectos nutricionais dos vinagres, eles contêm vitaminas, ácidos orgânicos (ácidos acéticos, fórmico, lático, málico, cítrico), proteínas e aminoácidos provenientes do fruto e da fermentação alcoólica. A qualidade final do vinagre e seus componentes nutricionais pode ser influenciada pela matéria-prima utilizada como substrato para sua fabricação, pelo sistema de acetificação utilizado e pela presença ou não de processos de envelhecimento em madeira (TESFAYE et al., 2002).

Os polifenóis são a classe de subprodutos da vinificação em maior quantidade no vinagre (VANIN et al, 2012). O estudo da presença de compostos fenólicos totais em vinagres de diferentes matérias-primas e a avaliação da capacidade antioxidante deles são importantes devido à crescente comercialização e consumo e à sua indicação constante como fontes de antioxidantes (KUSKOSKI et al., 2005; ÖZEN et al., 2020).

Embora essas propriedades funcionais e nutricionais dos vinagres não estejam totalmente esclarecidas devido à falta de um estudo profundo sobre este assunto, propalam-se seu efeito positivo no controle do $\mathrm{pH}$ do estômago para combater a gastrite, no ataque aos radicais livres, evitando a manifestação de certos tipos de cânceres. Outros efeitos são sua ação antisséptica e antibiótica, controle dos níveis glicêmicos e controle de pressão arterial (GALLO NETO, 2006).

O presente trabalho teve como objetivo avaliar a atividade antioxidante e determinar o conteúdo de compostos fenólicos em diferentes tipos de vinagre comercializados no mercado (maçã, vinho tinto, vinho branco e álcool). 


\section{MATERIAL E MÉTODOS}

\section{Amostras}

As amostras de vinagres foram obtidas no mercado varejista de Uberaba, MG, e São José do Rio Preto, SP, entre o período de julho e agosto de 2019. Foram avaliados 4 tipos de vinagres de 3 marcas diferentes (Tabela 1).

Tabela 1. Tipos e marcas de vinagres obtidos do comércio varejista de Uberaba, MG, e São José do Rio Preto, SP

\begin{tabular}{ccccc}
\hline Marcas & Maçã & Vinho tinto & Vinho branco & Álcool \\
\hline Marca 1 & A1 & B1 & C1 & D1 \\
Marca 2 & A2 & B2 & C2 & D2 \\
Marca 3 & A3 & B3 & C3 & D3 \\
\hline
\end{tabular}

Adicionalmente, foi utilizada nos ensaios de atividade antioxidante, uma solução de ácido acético $5 \%$, representando a concentração média desta substância presente nos vinagres comerciais.

As análises foram realizadas no Laboratório de Química do Instituto de Ciências Tecnológicas e Exatas (ICTE) da Universidade Federal do Triângulo Mineiro (UFTM) campus Uberaba.

\section{Análise de Compostos fenólicos totais}

A quantificação de compostos fenólicos totais foi determinada de acordo com o método de Singleton e Rossi (1965), usando o ácido gálico como padrão analítico. Para a quantificação de polifenóis foi primeiramente feita a curva padrão de ácido gálico $(0,25,50$, 100 e $150 \mathrm{mgmL}^{-1}$ ). Em cada tubo de ensaio devidamente identificado foram pipetados: $250 \mu \mathrm{l}$ da respectiva diluição do ácido gálico $+250 \mu \mathrm{L}$ do Reagente Folin-Ciocalteau +500 $\mu \mathrm{l}$ de carbonato de cálcio a $20 \%+4 \mathrm{~mL}$ de água destilada. O branco foi feito com $250 \mu \mathrm{L}$ de água no lugar da amostra. Após a agitação dos tubos, estes foram deixados em repouso por 2 horas. Com um espectrofotômetro UV visível a $765 \mathrm{~nm}$, pôde-se verificar a medida de absorbância. Com a construção de uma curva de calibração a partir dos dados de absorbância por concentração, foi possível obter uma equação de reta (1).

$$
y=a x+b
$$

Onde y representa a absorbância e $\mathrm{x}$ a concentração de compostos fenólicos. As leituras foram realizadas em triplicata e o resultado expresso por uma curva de $\mathrm{mg}$ de ácido gálico por litro de amostra.

Para o cálculo do teor de compostos fenólicos totais, em cada tudo de ensaio foram adicionados $250 \mu \mathrm{l}$ do vinagre $(1: 10)+250 \mu \mathrm{L}$ do Reagente Folin-Ciocalteau $+500 \mu \mathrm{L}$ de carbonato de cálcio a $20 \%+4 \mathrm{~mL}$ de água destilada. Preparou-se um branco com $250 \mu \mathrm{L}$ de água no lugar da amostra. Os tubos foram agitados e deixados em repouso por 2 horas. A absorbância foi medida em $765 \mathrm{~nm}$. O cálculo foi realizado substituindo o y da curva de calibração pelo valor da absorbância, e o valor de x obtido foi o teor de compostos fenólicos em $\mathrm{mg}$ de EAG (mg de equivalentes de ácido gálico) $\mathrm{mL}^{-1}$ de amostra. 


\section{Análise da atividade antioxidante pelo método $\mathrm{DPPH}^{\circ}$}

A atividade antioxidante da amostra nessa técnica foi medida através da sua capacidade em doar hidrogênio para o 2,2-diphenyl-1-picrylhydrazyl (DPPH') provocando a varredura deste radical livre e modificando a coloração da solução, ou seja, descoloração da solução de púrpura para amarelo.

A determinação da capacidade de varrer o radical livre estável DPPH' foi feita pelo método de Brand- Williams, Cuvelier e Berset (1995).

Preparou-se uma solução estoque de DPPH• com concentração de $40 \mu \mathrm{g} \mathrm{mL}^{-1}$. Uma alíquota de $0,3 \mathrm{~mL}$ da amostra de vinagre puro foi pipetada em tubo de ensaio juntamente com $2,7 \mathrm{~mL}$ da solução estoque de DPPH'. O branco foi preparado com $0,3 \mathrm{~mL}$ de solvente e 2,7 mL de metanol, enquanto no controle foram adicionados $0,3 \mathrm{~mL}$ de solvente e $2,7 \mathrm{~mL}$ de solução estoque de DPPH'. Os tubos foram agitados em vortex, deixados no escuro por 30 minutos e, em seguida, a leitura da absorbância foi feita em $517 \mathrm{~nm}$. O cálculo da atividade antioxidante em porcentagem foi realizado de acordo com a Equação (2).

$A A(\%)=\frac{A b s_{\text {controle }}-\text { Abs }_{\text {amostras }}}{A b s_{\text {controle }}} \times 100$

Onde $A b s_{\text {controle }}$ é a média das absorbâncias de controle e $A b s_{\text {amostras }}$, a média das absorbâncias da amostra. As medições foram realizadas em triplicata.

\section{Análise da atividade antioxidante pelo método ABTS ${ }^{\bullet+}$}

Neste método, a atividade antioxidante da amostra é medida através da sua capacidade em estabilizar o cátion radicalar $\mathrm{ABTS}^{++}$presente na solução, provocando a descoloração da solução e a diminuição da absorbância em $734 \mathrm{~nm}$. Neste caso, é necessário gerar a espécie estável $\mathrm{ABTS}^{*+}$ através de uma reação de oxidação do composto ABTS ${ }^{++}$utilizando perssulfato de potássio, por exemplo (BOROSKI et al., 2015). O antioxidante Trolox (6-hidroxi-2,5,7,8-tetrametilcromano-2-ácido carboxílico), análogo hidrossolúvel da vitamina $\mathrm{E}$, é usado como padrão neste ensaio e a reatividade das amostras é expressa como equivalentes molares deste padrão.

Seguindo o método proposto por Rufino et al. (2007) para gerar o radical ABTS ${ }^{\circ+}$ adicionou- se $5 \mathrm{~mL}$ da solução estoque de ABTS $(7 \mathrm{mM})$ em um balão volumétrico de 10 $\mathrm{mL}$, com $88 \mu \mathrm{L}$ da solução de persulfato de potássio $(140 \mathrm{mM})$. A mistura foi armazenada no escuro por 16 horas para o uso no dia da análise. Feito isso, diluiu-se $1 \mathrm{~mL}$ desta mistura preparada um dia antes em álcool etílico até obter uma absorbância de 0,70 nm $\pm 0,05 \mathrm{~nm}$ para o uso no mesmo dia da análise. Para a construção da curva de calibração foi elaborada a solução padrão de Trolox $(2.000 \mu \mathrm{M})$, com diluições variando de $50 \mu \mathrm{M}$ a $2000 \mu \mathrm{M}$.

Em um ambiente mais escuro, adicionou-se uma alíquota de $30 \mu \mathrm{L}$ de cada diluição de trolox, para os tubos de ensaio e misturou-se com $3,0 \mathrm{~mL}$ da solução do radical ABTS"+. A mistura foi homogeneizada em vortex e após 6 minutos foram realizadas as leituras das absorbâncias em $734 \mathrm{~nm}$. O álcool etílico foi utilizado como branco para calibrar o espectrofotômetro. As leituras foram realizadas em triplicata. Com as concentrações de Trolox $(\mu \mathrm{M})$ e as respectivas médias das absorbâncias, pode-se obter uma equação de reta através de regressão linear, de acordo com a Equação (3).

$y=-a x+b$ 
Em que $x$ é a concentração do Trolox $(\mu M)$ e y as respectivas médias das absorbâncias.

Para as amostras de vinagres de maçã, vinho tinto, vinho branco e álcool, utilizouse a quantidade de $30 \mu \mathrm{L}$ de cada amostra com $3,0 \mathrm{~mL}$ do radical $A B T S^{*+}$, adicionados nos tubos de ensaio e agitados no vortex. Depois de 6 minutos, foram realizadas as leituras das absorbâncias em $734 \mathrm{~nm}$. O álcool etílico foi utilizado como branco para calibrar o espectrofotômetro. As análises foram realizadas em triplicata.

Para o cálculo da capacidade antioxidante da amostra, substituiu-se o valor de absorbâncias no y da equação de reta da curva-padrão de Trolox obteve-se a concentração, que é x em $\mu \mathrm{M}$ de Trolox.

\section{Delineamento estatístico}

Os dados foram tabulados em planilha eletrônica, e os resultados submetidos à análise de variância (ANOVA), seguido do teste de Tukey a $5 \%$ de significância. Foram realizadas análises de correlação entre os dados de DPPH ${ }^{*}, \mathrm{ABTS}^{*+}$ e compostos fenólicos totais por meio de planilha eletrônicas.

\section{RESULTADOS E DISCUSSÃO}

\section{Compostos fenólicos totais}

A curva padrão de ácido gálico foi obtida através dos valores de concentração e absorbância em $765 \mathrm{~nm}$. Foi obtida uma equação da reta igual a $y=0,0064 x+0,0019$ com um $R^{2}=0,9998$ (Figura 1).

Figura 1. Curva padrão de ácido gálico

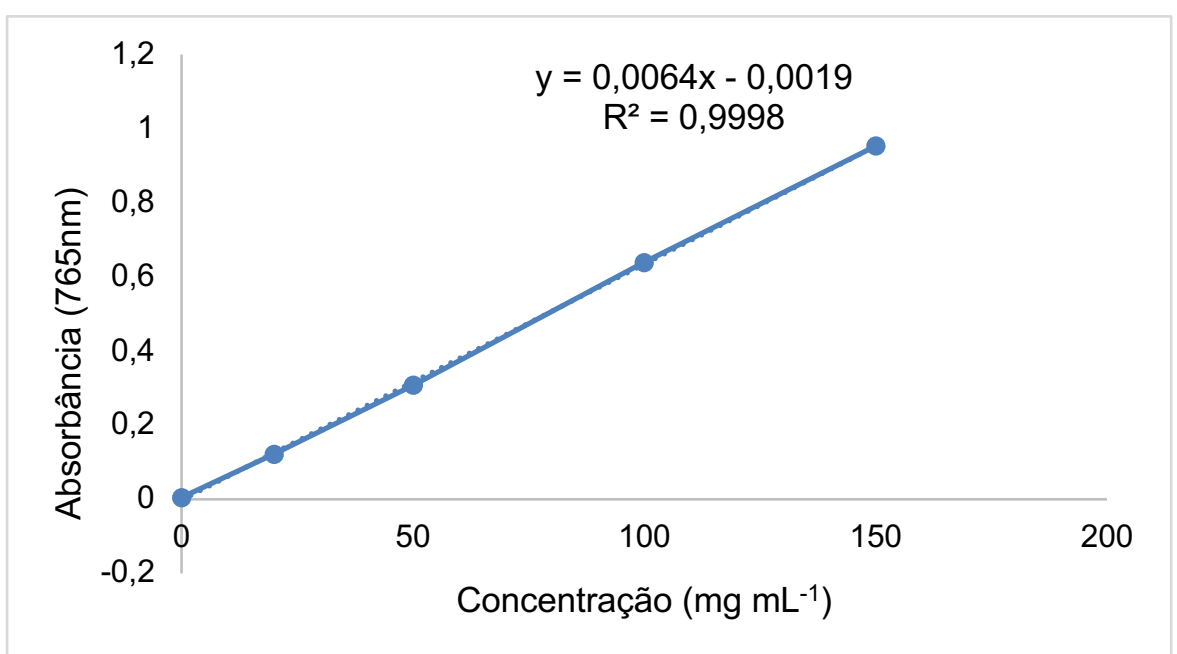

A partir da equação obtida pela curva padrão de ácido gálico determinou-se os teores de compostos fenólicos totais em mg EAGmL-1 de (Tabela 2).

Todas as amostras de vinagre demonstraram a presença de compostos fenólicos totais. Praticamente, todas as amostras apresentaram quantidades de compostos fenólicos diferentes entre si, mesmo entre os vinagres de mesmo tipo, mas de marcas diferentes. A 


\section{RB}

Revista Brasileira de Ciência, Tecnologia e Inovação

DOI: https://doi.org/10.18554/rbcti.v5i1.4358

única exceção foi o vinagre de álcool (D), no qual em todas as 3 marcas os valores foram iguais entre si (Tabela 2).

O vinagre de vinho tinto, que são as amostras B1, B2 e B3, apresentou teor maior de compostos fenólicos comparados aos outros vinagres. Por outro lado, os vinagres de álcool que são as amostras D1, D2 e D3 resultaram em valores muitos baixos de compostos fenólicos. Esse fato pode ser justificado pela diferença da matéria prima dos dois tipos de vinagre. A presença de polifenóis nas uvas escuras, matéria-prima do vinagre de vinho tinto, expressa uma significativa atividade antioxidante, o que explica seus altos valores. Já nos vinagres de álcool, possivelmente não existem compostos presentes, o que pode interferir no conteúdo de polifenóis.

Tabela 2. Teores de compostos fenólicos totais nos vinagres de maçã $(A)$ vinho tinto $(B)$, vinho branco (C) e álcool (D) de 3 marcas diferentes cada obtidas no mercado local

\begin{tabular}{cccc}
\hline Amostras & $\begin{array}{c}\text { Compostos fenólicos } \\
\text { totais- CFT }\left(\mathrm{mg} \mathrm{mL}^{-1}\right)\end{array}$ & Média das 3 marcas & $\begin{array}{c}\text { Ranking de maior } \\
\text { teor de CFT }\end{array}$ \\
\hline A1 & $291,51 \pm 11,93^{\mathrm{fg}}$ & & \\
\hline A2 & $302,97 \pm 7,16^{\text {ef }}$ & $304,88 \pm 9,97^{\mathrm{b}}$ & $2^{\circ}$ \\
\hline A3 & $320,16 \pm 10,82^{\mathrm{e}}$ & & \\
\hline B1 & $519,63 \pm 11,52^{\mathrm{b}}$ & & $1^{\circ}$ \\
\hline B2 & $587,34 \pm 15,07^{\mathrm{a}}$ & $516,34 \pm 13,99^{\mathrm{a}}$ & \\
\hline B3 & $442,03 \pm 15,39^{\mathrm{c}}$ & & $3^{\circ}$ \\
\hline C1 & $144,11 \pm 10,04^{\mathrm{d}}$ & & \\
C2 & $271,72 \pm 4,13^{\mathrm{g}}$ & $228,32 \pm 6,56^{\mathrm{c}}$ & $4^{\circ}$ \\
\hline C3 & $269,11 \pm 5,49^{\mathrm{g}}$ & & \\
\hline D1 & $20,16 \pm 1,56^{\mathrm{h}}$ & & \\
\hline D2 & $15,47 \pm 3,12^{\mathrm{h}}$ & $17,03 \pm 2,08^{\mathrm{d}}$ & \\
D3 & $15,47 \pm 1,56^{\mathrm{h}}$ & & \\
\hline
\end{tabular}

Médias obtidas das medidas analíticas em triplicata seguidas do desvio padrão.

Médias seguidas de mesma letra não deferem entre si pelo teste de Tukey $(p>0,05)$.

A- Vinagre de maçã; B- vinagre de vinho tinto; C- vinagre de vinho branco e D- vinagre de álcool.

Em estudo realizado com amostras de vinagre comercializado na região sul do país, resultados semelhantes foram encontrados nos teores de compostos fenólicos totais. Assim como neste trabalho, o vinagre de vinho tinto apresentou maior concentração de compostos fenólicos totais, $6,29 \mathrm{mg}$ de equivalente catequina em média, enquanto o vinagre de álcool, o menor valor, com 1,02 mg de equivalente catequina. Os vinagres de maçã e de vinho tinto apresentaram valores intermediários, usando a catequina como critério de referência. Neste trabalho, diferente do estudo dos autores, os vinagres de maçã e de vinho branco foram os que apresentaram valores intermediários (VANIN et al., 2012).

\section{Atividade Antioxidante pelo método DPPH'}

$\mathrm{Na}$ Tabela 3 encontram-se os resultados obtidos de atividade antioxidante de amostras de vinagres de diferentes marcas e tipos. 


\section{RB}

Revista Brasileira de Ciência, Tecnologia e Inovação

Tabela 3. Porcentagem da atividade antioxidante $\left(\mathrm{DPPH}^{\circ}\right)$ de amostras de vinagre comercial e ácido acético $5 \%$

\begin{tabular}{|c|c|c|c|}
\hline Amostras & Atividade antioxidante (\%) & Média das 3 marcas & Ranking \\
\hline $\mathrm{A} 1$ & $94,82 \pm 0,44$ ab & & \\
\hline $\mathrm{A} 2$ & $95,11 \pm 0,44^{a}$ & $95,04 \pm 0,46^{a}$ & $1^{0}$ \\
\hline A3 & $95,18 \pm 0,50^{a}$ & & \\
\hline B1 & $79,05 \pm 0,51^{\mathrm{e}}$ & & \\
\hline B2 & $81,52 \pm 0,54^{d}$ & $83,35 \pm 0,61^{b}$ & $2^{\circ}$ \\
\hline B3 & $89,49 \pm 0,77^{c}$ & & \\
\hline C1 & $92,88 \pm 0,62^{b}$ & & \\
\hline $\mathrm{C} 2$ & $95,62 \pm 0,22^{a}$ & $95,07 \pm 0,32{ }^{a}$ & $1^{\circ}$ \\
\hline C3 & $96,71 \pm 0,11^{a}$ & & \\
\hline D1 & $9,20 \pm 0,399$ & & \\
\hline D2 & $21,53 \pm 1,26^{f}$ & $12,93 \pm 0,76^{c}$ & $3^{\circ}$ \\
\hline D3 & $8,08 \pm 0,639$ & & \\
\hline Ácido acético 5\% & $3,37 \pm 1,35^{h}$ & $3,37 \pm 1,35^{d}$ & $4^{\circ}$ \\
\hline
\end{tabular}

Todas as amostras apresentaram atividade antioxidante. $O$ ácido acético e os vinagres de álcool (D1, D2 e D3), mostraram os menores valores (Tabela 3). Comparandose as amostras de vinagre de álcool, a marca D2 se destacou por ter mais atividade antioxidante. Isso pode ser explicado porque alguns aditivos contidos nas amostras podem ter influência sobre a atividade antioxidante, como exemplo, o metabissulfito de potássio e o dióxido de enxofre, que atuam como conservante e antioxidante. A quantidade de aditivos adicionada nas marcas D1 e D3 pode ser menor quando comparada à marca D2.

Os maiores valores de atividade antioxidante foram encontrados nos vinagres de maçã e de vinho branco, seguida pelo vinho tinto. Isso evidencia que os vinagres derivados de frutas apresentam maior atividade antioxidante quando comparados ao vinagre de álcool, possivelmente, devido à presença de compostos fenólicos (VANIN et al., 2012). Apesar de as amostras de vinagres de vinho tinto terem apresentado maiores teores de compostos fenólicos totais, estas não apresentaram valores mais expressivos para atividade antioxidante pelo método $\mathrm{DPPH}^{\circ}$. Isso se justifica pelo fato de que nem todos composto fenólico é bom doador de hidrogênio e, por este motivo, esta correlação nem sempre é observada.

As Figuras 2A e 2B apresentam as imagens dos tubos de ensaio contendo as amostras de vinagres e a solução estoque de $\mathrm{DPPH}^{\cdot}$ após os 30 minutos de repouso no escuro, ou seja, logo antes da leitura das absorbâncias em espectrofotômetro.

$\mathrm{Na}$ Figura 2B, observa-se que a coloração continua roxa, desse modo obtendo valores de atividade antioxidante baixos. De acordo com pesquisa de Ferreira (2015), os vinagres de maçã, vinho branco e vinho tinto apresentaram porcentagens de atividades antioxidantes significativamente iguais, com 96,06, 96,51 e 97,37\%, respectivamente. Já o vinagre de álcool apresentou $10,19 \%$ de poder redutor seguido do ácido acético $5 \% \mathrm{com}$ $6,67 \%$ de atividade antioxidante. Os resultados foram equivalentes aos obtidos pelos diferentes tipos de vinagres do presente estudo. 
Pela Figura 2A, pode-se ver a visível mudança de coloração de roxo para amarelo, comprovando os dados obtidos com maior porcentagem de atividade antioxidante.

Figura 2. Amostras de vinagres de maçã, vinho tinto e vinho branco (A) e amostras de vinagres de álcool, ácido acético $5 \%$ e controle (B) durante a determinação de atividade antioxidante pelo método DPPH•

(A)

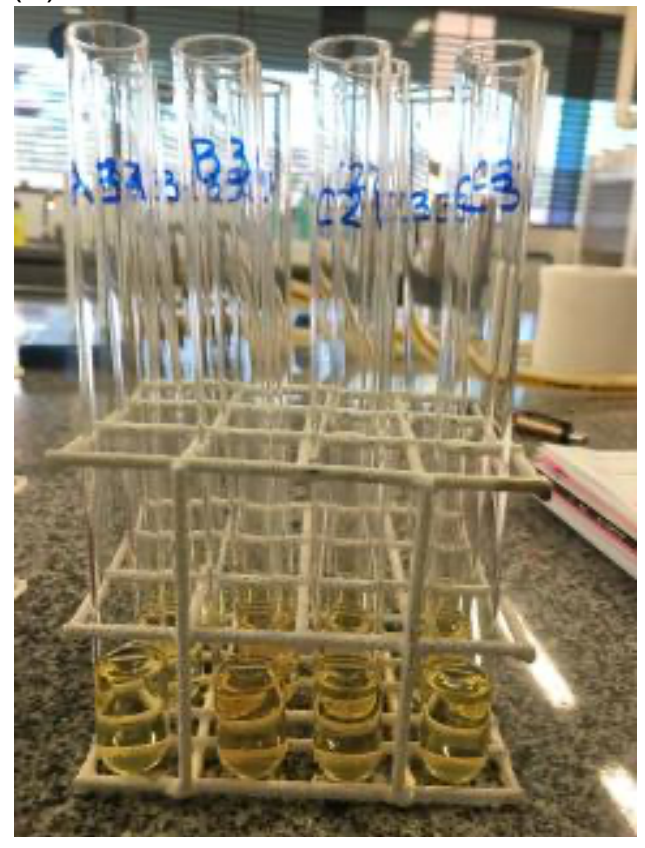

(B)

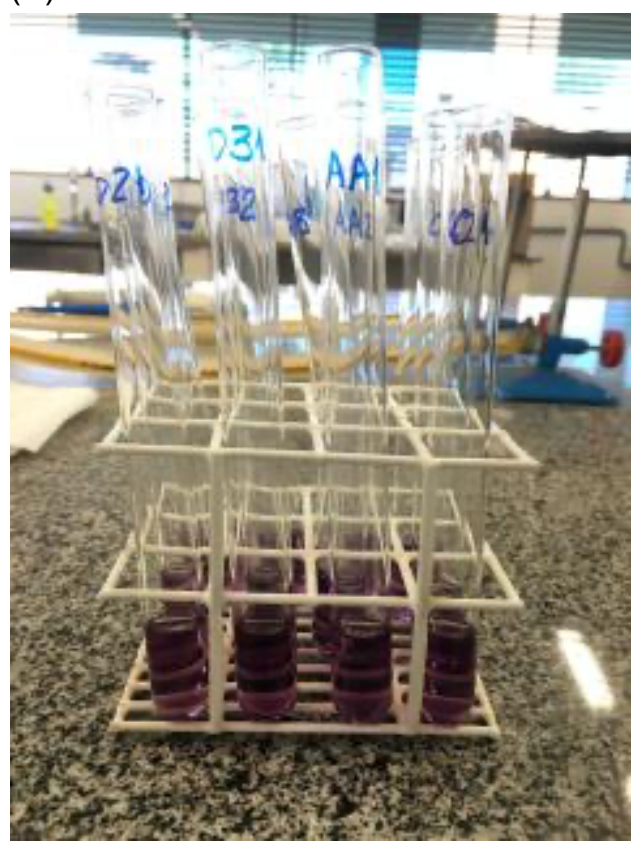

\section{Análise de atividade antioxidante pelo método ABTS ${ }^{+}$}

Primeiramente foi determinada a curva-padrão do Trolox (Figura 3). Obteve-se a equação de reta $y=-0,0003 x+0,6945$ com um $R^{2}=0,9972$ (Figura 3).

Figura 3. Curva-Padrão do Trolox

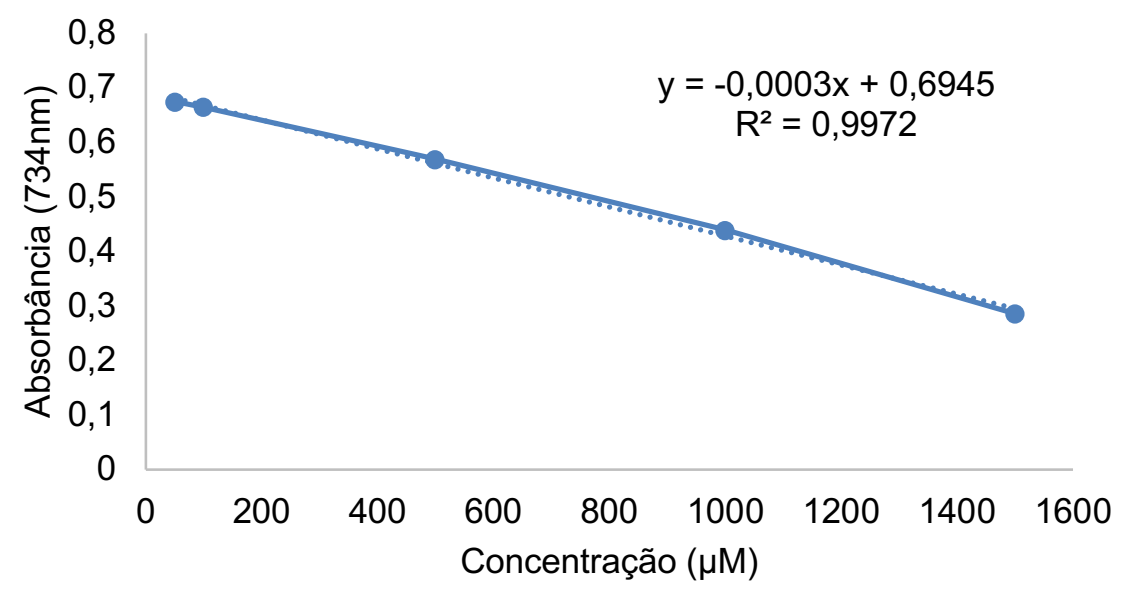

Obtida a equação que representa a curva padrão do Trolox, calcularam-se os valores 


\section{RB}

Revista Brasileira de Ciência, Tecnologia e Inovação

DOI: https://doi.org/10.18554/rbcti.v5i1.4358

de atividade antioxidante, isolando o valor de $x$ da equação da reta (Tabela 4).

Como observado, os valores de atividade antioxidante foram maiores nas amostras de vinho tinto (B1, B2 e B3), ou seja, os compostos capazes de inibir o radical ABTS"+ presentes em maiores quantidades.

Os três métodos realizados neste trabalho apresentaram correlações positivas. A correlação entre a análise de compostos fenólicos totais e DPPH e entre compostos fenólicos totais e $\mathrm{ABTS}^{\cdot+}$ foi de 0,69 e 0,97 , respectivamente, mostrando que quanto maior o teor de substâncias fenólicas, maior o poder antioxidante da amostra de vinagre.

Tabela 4. Valores de atividade antioxidante pelo método $\mathrm{ABTS}^{++}$de amostras de vinagre comercial e ácido acético $5 \%$

\begin{tabular}{cccc}
\hline Amostras & $\begin{array}{c}\text { Atividade antioxidante } \\
(\mu \text { M de Trolox })\end{array}$ & Média das 3 marcas & Ranking \\
\hline A1 & $1433,89 \pm 17,04^{\mathrm{d}}$ & \\
A2 & $1288,33 \pm 15,56^{\mathrm{e}}$ & $1468,70 \pm 17,78^{\mathrm{b}}$ & $2^{\circ}$ \\
A3 & $1683,89 \pm 20,74^{\mathrm{c}}$ & & \\
\hline B1 & $2295,00 \pm 0,00^{\mathrm{a}}$ & & \\
B2 & $2300,56 \pm 2,96^{\mathrm{a}}$ & $2215,74 \pm 10,62^{\mathrm{a}}$ & $1^{\circ}$ \\
\hline B3 & $2051,67 \pm 28,89^{\mathrm{b}}$ & & $3^{\circ}$ \\
\hline C1 & $608,33 \pm 26,67^{\mathrm{g}}$ & & \\
C2 & $807,22 \pm 45,19^{\mathrm{f}}$ & $1025,00 \pm 37,04^{\mathrm{c}}$ & $4^{\circ}$ \\
\hline C3 & $1659,44 \pm 39,26^{\mathrm{c}}$ & & \\
\hline D1 & $97,22 \pm 8,15^{\mathrm{h}}$ & & $5^{\circ}$ \\
\hline D2 & $109,44 \pm 12,59^{\mathrm{h}}$ & $94,26 \pm 11,11^{\mathrm{d}}$ & \\
\hline D3 & $76,11 \pm 12,59^{\mathrm{h}}$ & & \\
\hline Ácido acético $5 \%$ & $31,67 \pm 8,89^{\mathrm{h}}$ & $31,67 \pm 8,89^{\mathrm{e}}$ & \\
\hline
\end{tabular}

Médias obtidas das medidas analíticas em triplicata seguidas do desvio padrão.

Médias seguidas de mesma letra não deferem entre si pelo teste de Tukey $(p>0,05)$.

A- Vinagre de maçã; B- vinagre de vinho tinto; C- vinagre de vinho branco e D- vinagre de álcool.

Da mesma forma, os métodos DPPH' e $\mathrm{ABTS}^{++}$apresentaram correlação de 0,71. Esta correlação já era esperada, pois os dois métodos de atividade antioxidante apresentam mesmo mecanismo de ação, que se baseiam na transferência de átomos de hidrogênio pelas espécies antioxidantes para estabilizar radicais livres.

\section{CONCLUSÕES}

As amostras de vinagres comerciais de maçã, vinho tinto, vinho branco e de álcool apresentaram teor de compostos fenólicos e de atividade antioxidante. Os vinagres de vinho tinto foram as amostras com maiores teores de compostos fenólicos e de atividade antioxidante pelo método $A B T S^{+}$. Isso se deve ao fato de sua matéria-prima, a uva, ter presença de compostos que aumentam esse valor, como as antocianinas. Todas as amostras de vinagres analisadas pelo método $\mathrm{DPPH}^{\circ}$, também apresentaram atividade antioxidante. Destacaram-se os vinagres vinho branco e de maçã, seguido pelo vinagre de vinho tinto.

É de grande importância o estudo de compostos bioativos e atividade antioxidante de vinagres, por ser um produto muito consumido para realçar sabor. De acordo com o tipo 


\section{RB}

Revista Brasileira de Ciência, Tecnologia e Inovação

DOI: https://doi.org/10.18554/rbcti.v5i1.4358

de vinagre, as propriedades benéficas à saúde dos indivíduos podem ser variáveis, como comprovado nos testes in vitro deste estudo.

\section{REFERÊNCIAS}

ANAV. ASSOCIAÇÃO NACIONAL DAS INDÚSTRIAS DE VINAGRE. Os tipos de vinagres. 2019 Disponível em: <http://www.anav.com.br/clipping_interna.php?id=26>. Acesso em: 25 abr. 2019.

BOROSKI, M.; VISENTAINER, J. V.; COTTICA, S. M.; MORAIS, D. R. Antioxidantes: Princípios e métodos analíticos.1ed. Curitiba: Editora Appris, 2015.

BRAND-WILLIAMS, W.; CUVELIER, M. E.; BERSET, C. Use of a free radical method to evaluate antioxidant activity. LWT - Food Science and Technology, v. 28, n. 1, p. 25-30, 1995.

FERREIRA, A. C. F. Vinagre comercial: potencial inibitório da enzima alfa-amilase e atividade antioxidante. 2015. $41 \mathrm{f}$. Trabalho de Conclusão de Curso (Bacharelado em Biomedicina) - Universidade Federal do Triângulo Mineiro, Uberaba, MG, 2015.

GALLO NETO, G. N. Vinagre brasileiro ainda está distante do padrão de qualidade internacional. Jornal da Unicamp. Universidade Estadual de Campinas, 2006.

KUSKOSKI, E. M.; ASUERO, A. G.; TRONCOSO, A. M.; MANCINI-FILHO, J.; FETT, R. Aplicación de diversos métodos químicos para determinar actividad antioxidant en pulpa de frutos. Ciência e Tecnologia de Alimentos, v.25, n.4, p.726-732, 2005.

MARQUES, F. P. P.; SPINOSA, W.; FERNANDES, K. F.; CASTRO, C. F. S.; CALIARI, M. Padrões de identidade e qualidade de fermentados acéticos comerciais e vegetais.

Ciência e Tecnologia de Alimentos, v. 30, spl. 1, p. 119-126, 2010.

ÖZEN, M.; Özdemir, N., Filiz, B. E.; Budak, N. H.; Kök-Taş, T. Sour cherry (Prunus cerasus $\mathrm{L}$.) vinegars produced from fresh fruit or juice concentrate: bioactive compounds, volatile aroma compounds and antioxidant capacities. Food Chemistry, v. 309, n. 30, 2020.

RIZZON, L. A. Elaboração de vinagre. Bento Gonçalves: Embrapa Uva e Vinho, 2002.

RUFINO, M. S. M.; ALVES, R. E.; BRITO, E. S.; MORAIS, S. M.; SAMP, C. G. et al. Metodologia Científica: Determinação da Atividade Antioxidante Total em Frutas pela Captura do Radical Livre ABTS ${ }^{+}$. Fortaleza: Embrapa Agroindústria Tropical, 2007. 4p. (Comunicado Técnico on-line, 128).

SINGLETON, V. L.; ROSSI JR, J. A. Colorimetry of total phenolics with phosphomolybdicphosphotungstic acid reagents. American Journal of Enology and Viticulture, v.16, n. 3, p. 144-158, 1965. 
TESFAYE, W. M.; MORALES, M. L.; GARCÍA-PARRILLA, M. C.; TRONCOSO, A. M. Wine vinegar: technology, authenticity and quality evaluation. Trends in Food Science and Technology, v. 13, n. 1, p. 12-21, 2002.

VANIN, A.; RUFFATO, S. R.; SIVIERO, J.; SALVADOR, M. Atividade antioxidante e perfil fenólico de diferentes tipos de vinagres comercializados na região Sul do Brasil.

Alimentos e Nutrição, v. 23, n. 2, p. 251-257, 2012. 